\title{
LES DÉMENCES EN AFRIQUE SUBSAHARIENNE : ASPECTS CLINIQUES ET ÉTIOLOGIQUES EN MILIEU HOSPITALIER À OUAGADOUGOU (BURKINA FASO)
}

\section{DEMENTIAS IN SUB-SAHARAN AFRICA: CLINICAL AND ETIOLOGICAL ASPECTS IN HOSPITAL ENVIRONMENT IN OUAGADOUGOU (BURKINA FASO)}

NAPON Christian 1

TRAORE S. Idris S. 1

NIAKARA Ali ${ }^{2}$

OUANGO G. Jean-Gabriel ${ }^{3}$

KABRÉ Abel ${ }^{4}$

KABORE Jean ${ }^{1}$

1. Service de Neurologie, CHU Yalgado Ouédraogo, Ouagadougou, Burkina Faso

2. Service de Cardiologie, CHU Yalgado Ouédraogo, Ouagadougou, Burkina Faso

3. Service de Psychiatrie, CHU Yalgado Ouédraogo, Ouagadougou, Burkina Faso

4. Service de Neurochirurgie, CHU Yalgado Ouédraogo, Ouagadougou, Burkina Faso

E-Mail Contact - NAPON Christian : cnapon (at) gmail (dot) com

Mots-clés: Afrique, Alzheimer, , Burkina Faso, Démence

Keywords: Africa, Alzheimer, Burkina Faso, Dementia

\section{RESUME}

\section{Description}

En Afrique subsaharienne, l'amélioration progressive des conditions de vie a pour corollaire l'émergence accrue de certaines pathologies en rapport avec l'âge dont la démence. L'objectif de cette étude était de décrire les différents aspects des démences au Centre Hospitalier Universitaire Yalgado Ouédraogo.

\section{Méthodes}

C'est une étude transversale qui a inclus pendant deux ans les sujets âgés de plus de 15 ans hospitalisés ou ayant consulté dans les services de Neurologie, Psychiatrie, Cardiologie et Neurochirurgie et répondant aux critères diagnostiques de démence du DSM-IV.

\section{Résultats}

Soixante-douze démences ont été diagnostiquées soit une prévalence hospitalière de 4,55 pour mille patients et 2,21 \% des patients hospitalisés. L'âge moyen était de 62,20 ans avec un sex ratio de 2 . L'installation des troubles cognitifs et comportementaux a été insidieuse et progressive dans la plupart des cas. La démence était sévère avec un score MMS inférieur à 9 dans la majorité des cas. Les démences secondaires dites curables étaient les plus fréquentes $(68,05 \%)$, dominées par les démences vasculaires et les causes neurochirurgicales. Seulement $18 \%$ des démences étaient dégénératives avec à leur tête les démences de type Alzheimer. L'entourage familial reste fortement impliqué dans la démarche de soins et la prise en charge.

\section{Conclusion}

La démence est une réalité au Burkina Faso, avec une prévalence sous-estimée. Il est donc nécessaire de mener des études en population dans le but d'envisager des mesures préventives et de prise en charge adaptées à notre contexte. 


\section{SUMMARY}

\section{Background}

In sub-Saharan Africa, the gradual improvement of living conditions has for corollary the emergence of some diseases related to age of which dementia.

\section{Objective}

The objective of this study was to describe the various aspects of dementia at the Teaching Hospital Yalgado Ouédraogo.

\section{Methodology}

It was a cross-sectional study that included two years for people aged over 15 , hospitalized or consulting services in Neurology, Psychiatry, Cardiology and Neurosurgery and meets the diagnostic criteria of dementia of DSM-IV.

\section{Results}

Seventy-two were diagnosed with dementia or a hospital prevalence of 4.55 patients per thousand and $2.21 \%$ of hospitalized patients. The average age was 62.20 years with a sex ratio of 2 . The installation of cognitive and behavioural disorders has been insidious and gradual in most cases. Dementia was severe with a MMS score less than 9 in most cases. The so-called secondary curable dementias were the most frequent $(68.05 \%)$, dominated by vascular dementia and neurosurgical cases. Only $18 \%$ of degenerative dementias were headed with dementia of Alzheimer type. The family remains deeply involved in the process of care and support.

\section{Conclusion}

Dementia is a reality in Burkina Faso, with a prevalence underestimated. It is therefore a need for population studies in order to consider preventive measures and care tailored to our context.

\section{INTRODUCTION}

En Afrique subsaharienne, l'amélioration progressive des conditions d'existence s'accompagne d'une augmentation substantielle de l'espérance de vie. Ceci a pour corollaire l'émergence accrue de certaines pathologies en rapport avec l'âge dont la démence. Pathologie surtout du sujet âgé, elle traduit une dégradation progressive des fonctions cognitives, entravant l'autonomie dans la vie quotidienne [1]. Sa prévalence est de plus en plus élevée avec le vieillissement de la population. En effet, on estimait à 25 millions le nombre de personnes atteintes de démences dans le monde en 2000 [22]. En revanche on dispose de très peu de données épidémiocliniques sur les démences en Afrique (Nigeria, Egypte, Afrique du Sud, Sénégal) et particulièrement au Burkina Faso. C'est dans ce contexte qu'a été menée cette étude dont l'objectif était d'analyser les différents aspects de cette pathologie afin de contribuer à apporter des réponses adaptées à notre contexte.

\section{POPULATION ET METHODES}

\section{Nature de l'étude}

II s'agit d'une étude transversale réalisée du 1er Mars 2005 au 28 Février 2007 dans les services de Neurologie, Psychiatrie, Neurochirurgie et Cardiologie du CHU Yalgado Ouédraogo de Ouagadougou au Burkina Faso.

\section{Critères d'inclusion et d'exclusion}

L'inclusion a été systématique parmi les consultants et les hospitalisés. L'étude a concerné les patients répondant aux critères suivants :

- âge supérieur ou égal à 15 ans ;

- être hospitalisé ou consulter dans les services de Neurologie, Psychiatrie, Neurochirurgie et cardiologie du CHU Yalgado Ouédraogo ;

- répondre aux critères diagnostics de démence du DSMIV avec des troubles dont la durée d'évolution était d'au moins six mois ;

- avoir des parents ou un entourage consentant à participer à l'étude.

Ont été exclus les pseudo-démences, les épisodes confusionnels et les psychoses chroniques. 


\section{Echantillonnage}

Dans un premier temps, les patients présentant une plainte mnésique ou d'autres symptômes comportementaux ou psychologiques évocateurs étaient soumis à une évaluation de leurs fonctions cognitives à travers divers outils (MMSE [5], IADL (Instrumental Activities of Daily Living : ce test contient des échelles d'activités de la vie courante que sont la capacité à utiliser le téléphone, les moyens de transport, la responsabilité pour la prise des médicaments et la capacité à gérer son budget) [3], fluence verbale (mesure du nombre de mots énoncés dans une catégorie donnée dans un temps limité de 5 minutes) [17], test de l'horloge [14]. A l'issue de cette première évaluation, le diagnostic de démence était fait sur la base des critères du DSM IV [12]. Les patients ne remplissant pas ces critères étaient réévalués dans un délai de six mois après un traitement adapté (antidépresseur, antiépileptique, rééquilibration hydro électrolytique...). La sévérité de la démence était appréciée par le score obtenu au MMSE [5]. Dans un second temps, en fonction des éléments d'orientation étiologique, un bilan clinique plus approfondi (Echelle de dysfonctionnement frontal...) était pratiqué ainsi qu'un bilan paraclinique pour déterminer l'étiologie de la démence, en utilisant des critères pour le diagnostic de certaines étiologies [12]. Les démences dont l'étiologie n'a pu être déterminée étaient classées "démence sans précision ». Dans tous les cas, le diagnostic a été posé de manière prudente, après des entretiens répétés avec le malade et sa famille, et une surveillance prolongée de l'évolution (six mois).

\section{Analyse statistique des données}

Les données ont été recueillies à l'aide de fiches individuelles de collecte, vérifiées, classées et conservées sous forme de fiches informatiques et analysées à l'aide du logiciel Epi info version 3.3.2. Le test du Chi carré, le test de Fisher exact et le test de Kruskall-Wallis pour deux groupes ont servi à la comparaison des données avec un seuil de significativité de 0,05.

\section{RESULTATS}

\section{Prévalence et caractéristiques socio-démographiques}

Au total, sur les 15815 patients qui ont consulté dans les services d'étude, 72 cas de démences ont été diagnostiquées soit une prévalence de 4,55 pour 1000. Durant cette même période, 2396 patients ont été hospitalisés dans l'ensemble des services d'étude dont 53 sujets déments $(73,61 \%$ des cas de démences) soit une prévalence de $2,21 \%$ des hospitalisés. L'hospitalisation était significativement associée à une plus grande fréquence des troubles du comportement $(p=0,019)$, du déficit moteur $(p=0,017)$ et de l'altération de l'état général $(p=0,02)$. L'âge moyen des patients était de 62,20 ans et la tranche d'âge de 60 à 70 ans représentait $27,8 \%$ de l'échantillon avec un sex ratio de 2 . Quarante-sept virgule vingt-deux pour cent des patients étaient des cultivateurs et femmes au foyer et $51,40 \%$ étaient illettrés.

\section{Aspects cliniques}

Dans $81,94 \%$ des cas, l'entourage avait été à l'origine de la demande de soins. La demande émanait de l'entourage beaucoup plus dans les démences sévères que dans les démences légères $(p=0,001)$. Celle-ci était motivée par les troubles du comportement dans $48,61 \%$ des cas, des troubles mnésiques dans 27,78 $\%$ des cas et une altération de l'état général dans $26,39 \%$ des cas. Les antécédents étaient constitués par une :

- une HTA chez $34,72 \%$ des patients, évoluant depuis en moyenne 10 ans. Elle était beaucoup plus fréquente dans les démences vasculaires $(63,20 \%)$ que dans les démences dégénératives $(28,60 \%)$.

- un accident vasculaire cérébral chez $29,17 \%$ qui était présent chez 52,60 \% des démences vasculaires.

- et une dépression chez $13,89 \%$.

Les autres antécédents neurologiques étaient représentés par un traumatisme crânien grave dans 19,44\% des cas et une infection du système nerveux central dans $13,89 \%$ des cas.

Les troubles cognitifs étaient d'installation insidieuse et d'évolution lentement progressive dans $75 \%$ des cas avec une moyenne de 22,83 mois. La sémiologie cognitive était constituée par : - une atteinte de la mémoire sémantique dans $59,30 \%$ des cas, - une aphasie amnésique avec manque du mot dans $43,55 \%$ des cas, $\begin{array}{llcccc}\text { - une } & \text { apraxie } \begin{array}{c}\text { constructive } \\ \text { - une }\end{array} \text { agnosie } & 55,56 & \% & \text { des } & \text { chez }\end{array}$

- des troubles des fonctions exécutives et du jugement dans $77,75 \%$ des cas.

Le ralentissement psychomoteur, l'anxiété et l'apathie étaient les symptômes psychologiques présentés par $78,79 \%, 65,15$ et $57,58 \%$ des patients respectivement. Quatre-vingts treize virgule quatre-vingts quatorze pour cent des patients avaient des troubles du comportement, avec des fugues dans $12,51 \%$ des cas. Les troubles des conduites élémentaires étaient constitués par des troubles du sommeil dans $64,64 \%$ des cas, 
des troubles des conduites alimentaires dans $12,12 \%$ des cas. Plus du tiers des patients présentait un déficit moteur et le quart était dénutri et déshydraté. L'évaluation psychométrique a permis de noter que la majorité de nos patients avaient une démence sévère avec un score MMS inférieur à $9(73,61 \%$ des cas). Seulement 18 cas de démences légères soit $25 \%$ des patients étaient retrouvés. Soixante-dix neuf pour cent d'entre eux étaient dépendants de l'entourage pour les activités de la vie quotidienne (capacité à utiliser le téléphone, les moyens de transport, responsabilité pour la prise des médicaments et capacité à gérer son budget).

\section{Données paracliniques}

La tomodensitométrie (TDM) cérébrale réalisée chez 52 patients était normale dans 4 cas. Elle montrait une atrophie cortico-sous-corticale dans $40,38 \%$ des cas et des images de leucoencéphalopathie dans $17,31 \%$ des cas (Tableau I). L'électroencéphalogramme (EEG) n'a été réalisée que chez 9 patients et montrait des signes de souffrance cortico-sous-corticale diffuse dans 5 cas. Le bilan biologique montrait surtout des troubles métaboliques dans 22 cas (troubles hydroélectrolytiques avec notamment des hypernatrémies par déshydratation extracellulaire et des hypoprotidémies responsables d'état de dénutrition). La sérologie syphilitique (TPHA/VDRL) était positive chez deux patients dans le sang et le LCR et 3 patients étaient VIH 1 positif.

\section{Aspects nosologiques}

La répartition des patients selon le type de démence est représentée par le tableau II. Les étiologies des démences dégénératives étaient représentées par la maladie d'Alzheimer dans 7 cas (9,72 \%), les démences frontotemporales dans 3 cas $(4,16 \%)$ et la maladie de Parkinson dans 2 cas $(2,77 \%)$. II a été noté un syndrome démentiel chez un patient atteint de chorée de Huntington évolutive. Les cas de démences dites curables étaient au nombre de 49 (soit 68,05 \%). Elles étaient vasculaires dans $51,35 \%$ des cas de démences curables, de cause neurochirurgicale dans $32,43 \%$ des cas et infectieuse dans $10,81 \%$ des cas. Un cas de démence post-traumatique a été observé. Soixante-dix virgule quatre-vingts pour cent des patients avaient au moins une autre pathologie associée à la démence. La co-morbidité était significativement liée à un âge plus avancé $(p=0,02)$. Il s'agissait d'une cardiopathie dans $23,61 \%$ des cas et d'une épilepsie dans $6,94 \%$ des cas. Deux patientes présentaient une ostéoporose. Ailleurs, il s'agissait d'affections respiratoires, uro-génitales et infectieuses.

\section{Acteurs de la prise en charge et évolution}

Lorsque l'entourage intervenait dans la prise en charge, il était représenté par les descendants dans 76,39 \% des cas. Dans $25 \%$ des cas, un membre de la famille avait été choisi pour s'occuper en permanence des soins corporels du patient. L'évolution au cours de la période d'étude a été marquée par le décès de $11,11 \%$ des patients et, $37,50 \%$ ont été perdus de vue.

\section{DISCUSSION}

Le contexte socioculturel qui fait de la maladie du sujet âgé une fatalité, le cadre d'étude qui est le centre hospitalier national de référence (dernier recours de la pyramide sanitaire) et l'inaccessibilité de certains examens ont été les limites de cette étude. Par ailleurs, la rareté des études africaines sur le sujet ne permet pas toujours de faire des comparaisons. Un autre problème est lié à l'utilisation de l'instrument MMSE qui est inadapté pour nos populations qui sont surtout illettrées mais aussi la validité externe des résultats obtenus. La relative faible prévalence dans nos pays [2, 6, 23] par rapport aux pays européens [19] pourrait s'expliquer d'une part par le contexte socioculturel qui fait des troubles mnésiques du sujet âgé une fatalité liée à l'âge et la faible détection des troubles mnésiques par les praticiens [9], et d'autre part par la relative plus courte espérance de vie [7].

La majorité des patients a été vue en hospitalisation (73,61\%). Celle-ci était liée à la consultation tardive, à un moment où le patient présente des signes et symptômes négatifs et/ou invalidants. En effet, l'hospitalisation était significativement associée à une plus grande fréquence des troubles du comportement $(p=0,019)$, du déficit moteur $(p=0,017)$ et de l'altération de l'état général $(p=0,02)$.

L'âge moyen des patients était de 62 ans avec une prédominance de la tranche de 60 à 70 ans. Ces résultats sont comparables à ceux d'Avodé au Bénin [2]. Ils sont cependant différents de ceux de Ska au Canada qui notait une plus grande fréquence après 75 ans [19] et de Lechowski en France qui trouvait un âge moyen de 77,8 ans avec une prédominance des sujets de plus de 80 ans [10]. Cette différence pourrait s'expliquer par l'espérance de vie beaucoup plus courte dans les pays en voie de développement comme le nôtre où la fréquence et la gravité des pathologies du sujet âgé augmentent avec l'âge, emportant les plus vieux et réduisant ainsi leur effectif. Le sex ratio était de 2. Harvey en Grande Bretagne notait aussi une prédominance masculine à 58\% [7]. Par contre, Avodé au Benin [2], Grunitzky au Togo [6] et Lindsay au 
Canada [13] notaient une prédominance féminine. Letenneur en France [11] notait une prédominance masculine avant 80 ans et une prédominance féminine après cet âge et concluait à une interaction possible entre l'âge et le sexe.

Les cultivateurs et femmes au foyer représentaient $47,22 \%$ de notre échantillon. Cela concorde ave la distribution socioprofessionnelle de la population burkinabè qui est à $88 \%$ éleveur et agriculteur. Par ailleurs, les retraités consulteraient beaucoup plus dans des structures qui leurs sont réservées.

La moitié de nos patients étaient illettrés $(51,4 \%)$. Cela pourrait s'expliquer d'une part par le faible taux de scolarisation qu'a connu notre pays par le passé. D'autre part, le bas niveau de scolarisation serait un facteur de risque de démence selon Letenneur et Lindsay $[11,13]$. L'entourage était à l'origine de la demande de soins dans $81,94 \%$ des cas. Mouanga au Congo, dans son étude sur le profil des patients observés en consultation externe de psychiatrie notait aussi que la consultation était suscitée par d'autres personnes dans $18,5 \%$ des cas [15]. Cela traduit sans doute l'implication du groupe familial dans la démarche de soins dans notre contexte. Par ailleurs l'entourage interviendrait dans cette démarche à un stade tardif, après avoir épuisé les recours traditionnels et extra institutionnels. En effet, la demande émanait de l'entourage beaucoup plus dans les démences sévères que dans les démences légères $(p=0,001)$. Les troubles du comportement $(48,61 \%)$, l'altération de l'état général $(26,39 \%)$ et le déficit moteur $(33,33 \%)$ ont été les motifs de consultation les plus fréquents. Ils sont le reflet du retard à la consultation et ont été retrouvés significativement liés à l'hospitalisation (p égal à 0,$019 ; 0,017$ et 0,02 respectivement).

Présente chez $34,72 \%$ des patients, l'HTA était beaucoup plus fréquente dans les démences vasculaires $(63,20 \%)$ que dans les démences dégénératives $(28,60 \%)$. Quant aux accidents vasculaires cérébraux, ils faisaient partie des antécédents retrouvés chez les patients atteints de démence vasculaire $(52,60 \%)$. Tsuchihashi notait dans l'étude d'Hisayama que l'HTA et la survenue d'un AVC constituaient des facteurs de risque significatif pour la démence vasculaire [21]. Cependant il soulignait l'implication possible de ces facteurs dans la pathogenèse de la maladie d'Alzheimer.

L'atteinte mnésique était sémantique dans $59,30 \%$ des cas. Les troubles sémantiques sont le reflet de la consultation tardive car s'observent le plus souvent à un stade avancé de l'évolution de la démence. Ils expliquent la prédominance de l'aphasie amnésique avec manque du mot juste sur les autres troubles du langage. Etaient aussi associés une apraxie surtout constructive, une agnosie ainsi que des troubles des fonctions exécutives et du jugement dans $77,75 \%$ des cas.

Les symptômes psychologiques étaient dominés par le ralentissement psychomoteur, l'anxiété, les troubles de l'identification des proches et l'apathie. Pour Thomas, l'apathie serait le trouble le plus fréquent parmi les symptômes psychologiques de la maladie d'Alzheimer [20]. Une notion de fugues a été notée chez $12,51 \%$ des patients. Avodé et Rolland ont retrouvé des proportions comparables [2,18]. Elles étaient liées à une sévérité beaucoup plus grande du déclin cognitif et à une dépendance beaucoup plus importante.

Le quart des déments était déshydraté et dénutri avec des troubles métaboliques à la biologie. Les incapacités pour les activités de la vie quotidienne augmentant avec l'évolution clinique de la démence, les patients finissent par être plus ou moins dépendants de l'entourage, y compris pour leur alimentation. Et lorsque les apports sont insuffisants par rapport aux besoins physiologiques, s'installe progressivement un déséquilibre qui abouti à terme, lorsqu'il n'est pas corrigé, à une déshydratation et/ou une dénutrition plus ou moins sévères.

La majorité de nos patients avaient une démence sévère avec un score MMS inférieur à 9 . Seuls $21 \%$ des patients avaient une autonomie satisfaisante. Pour Barberger-Gateau [4], la démence est un déterminant majeur de la perte d'autonomie fonctionnelle des personnes âgées. L'atrophie cortico-sous-corticale était l'anomalie scanographique la plus observée, surtout dans les démences dégénératives. Toutefois, comme le soulignait Patterson, la neuroimagerie n'est pas très efficace pour distinguer la maladie d'Alzheimer ou d'autres démences corticales du processus normal du vieillissement [16]. Son objectif est d'exclure les étiologies secondaires potentiellement réversibles telles les lésions intracérébrales (tumeur, hématome sousdural chronique) ou l'hydrocéphalie à pression normale.

L'EEG quand elle est réalisée montrait surtout une souffrance cortico-sous-corticale diffuse. Elle reste peu contributive sauf dans certaines étiologies telles la maladie de Creutzfeld-Jacob et les épilepsies [8]. Contrairement aux données européennes [4,7], les démences secondaires ont été plus fréquemment retrouvées ; elles représentaient $68,05 \%$ des démences. Cette nosographie serait liée à la jeunesse des populations dans notre contexte où l'espérance de vie, quoique croissante demeure encore faible comparativement à celle des pays développés. Par ailleurs, dans notre contexte socioculturel où les troubles mnésiques et comportementaux du sujet âgé bénéficient d'une prise en charge et d'une protection sociale longtemps efficace (entourage, famille), la consultation n'est le plus souvent motivée que par des signes invalidants, le plus souvent symptomatiques de l'étiologie de la démence.

Elles étaient dominées par les démences vasculaires qui représentaient $26,39 \%$ de l'ensemble des démences et les affections neurochirurgicales. Nous avons par ailleurs noté un cas de paralysie générale syphilitique tardive confirmée par la biologie et un cas de démence post traumatique. 
Les étiologies des démences dégénératives étaient représentées par la maladie d'Alzheimer dans 7 cas, les démences frontotemporales dans 3 cas et la maladie de Parkinson dans 2 cas. II a été noté un syndrome démentiel chez un patient atteint de Chorée de Huntington évolutive. Plus de deux tiers (2/3) des patients avaient au moins une autre pathologie associée à la démence. Cette co-morbidité était significativement liée à un âge plus avancé $(p=0,02)$. Cela pourrait être dû aux défaillances viscérales multiples qui peuvent s'installer avec l'âge. Les pathologies cardiaques, respiratoires, uro-génitales et infectieuses étaient plus fréquemment associées. L'entourage intervenait dans la prise en charge dans la quasi-totalité des cas. Dans $25 \%$ des cas, un membre de l'entourage avait été désigné pour s'occuper en permanence des soins corporels et des besoins du dément. Lindsay au Canada notait que $94 \%$ des soignants des personnes démentes étaient des membres de la famille [13]. Le recours à une structure sanitaire n'intervient que lorsque l'état clinique précaire des patients se décompense ou quand l'entourage est débordé.

L'issue a été fatale dans $11,11 \%$ des cas. Barberger-Gateau trouvait que le décès chez les déments était le plus souvent attribué aux maladies cardiovasculaires, respiratoires, mentales (cérébrales), nutritionnelles et neurologiques qui sont souvent associées [4].

\section{CONCLUSION}

La démence, longtemps considérée comme inexistante dans les pays sous-développés, est de nos jours une réalité quoique encore mal connue. Sa prévalence reste sous-estimée. Cette étude a permis de trouver une prévalence hospitalière de 4,55 pour mille patients. Elle survient beaucoup plus souvent chez des sujets relativement jeunes, de sexe masculin, résidant en milieu urbain, illettrés, cultivateurs ayant une HTA. Vu la transition démographique qui est en train de s'opérer dans nos pays, il convient de mener des études beaucoup plus élargies, en population, qui permettraient de mieux cerner le problème et d'envisager des solutions et des axes de prévention adaptés à notre contexte.

Tableau I : Répartition des 52 patients selon les principaux aspects TDM (Table I: Distribution of the $\mathbf{5 2}$ patients according to the main C. T. scans aspects)

\begin{tabular}{|l|l|l|}
\hline \multicolumn{1}{|c|}{ Aspect TDM } & Nombre & Pourcentage \\
\hline Atrophie cortico-sous-corticale & 21 & 40,38 \\
\hline Leucoencéphalopathie vasculaire & 9 & 17,31 \\
\hline Hématome sous-dural chronique & 5 & 9,62 \\
\hline Hydrocéphalie chronique & 4 & 7,69 \\
\hline Encéphalite & 4 & 7,69 \\
\hline
\end{tabular}

Tableau II : Répartition des étiologies des démences dites curables. (Table II: Distribution of the aetiologies of the curable dementias)

\begin{tabular}{|l|l|l|}
\hline \multicolumn{1}{|c|}{ Étiologie } & Nombre & Pourcentage \\
\hline Démence vasculaire & 19 & 51,35 \\
\hline Causes neurochirurgicales & 12 & 32,43 \\
\hline - Hématome sous-dural chronique & 5 & 13,51 \\
\hline - Hydrocéphalie à pression normale & 4 & 10,81 \\
\hline - Tumeur intracrânienne & 3 & 8,11 \\
\hline Causes infectieuses & 4 & 10,81 \\
\hline Intoxication éthylique & 1 & 2,70 \\
\hline Démence post-traumatique & 1 & 2,70 \\
\hline
\end{tabular}




\section{REFERENCES}

1. AURIACOMBE S, ORGOGOZO JM. Syndrome démentiel. Encycl Méd Chir, Neurologie 17-044-C40, 2004, 1-7.

1. AVODE DG, GANDAHO P, DA-CRUZ PC, AHYI RG, ZOHOUN TH. Les démences séniles en milieu hospitalier à Cotonou. Le Bénin Médical 1998;7:23-27.

2. BARBERGER-GATEAU P, FABRIGOULE C, HELMER C, ROUCH I, DARTIGUES JF. Functional impairement in instrumental activities of daily living: an early clinical sign of dementia. J Am Geriatr Soc. 1999; 47:456-462.

3. BARBERGER-GATEAU et al. The contribution of dementia in the disablement process and modifying factors.Dement Geriatr Cogn Disord 2004;18:330-7.

4. DÉROUESNÉ C, POITRENAUD J, HUGONOT C, KALAFAT M, DUBOIS B, LAURENT B. « le Mini Mental State Examination (MMSE) : un outil pratique pour l'évaluation de l'état cognitif des patients pour le clinicien». Presse Méd. 1999;28:1141-1148.

5. GRUNITZKY et al. Prévalence des principales affections neurologiques en milieu rural au Togo. Neurologie tropicale, Paris 1993;4:13-16.

6. HARVEY RJ, SKELTON-ROBINSON M, ROSSOR MN. The prevalence and causes of dementia in people under the age of 65 years. J Neurol Neurosurg Psychiatry 2003; 74:1206-1209.

7. HIRSH E, MATON B, KURTZ D. Bases neurophysiologiques de l'électroencéphalographie clinique et principales indications. Encycl Méd Chir, Neurologie, 17-031-A-10, 1995, 11p.

8. JORAY S, PERTOLDI W, BULA C. Detection of cognitive disorders in clinical practice. Rev Med Suisse Romande 2000; 120:847-52.

9. LECHOWSKI L, HARBOUN M, DIEUDONNE B, HERNANDEZ K, TORTRAT D, FORETTE B, TEILLET L, VELLAS B. Clinical features of ambulatory patients over 80 years of age followed for Alzheimer's disease. French prospective multicenter study REAL.FR. Rev Med Interne. 2003; 24:307-313.

10.LETENNEUR L, GILLERON V, COMMENGES D, CHELMER, ORGOGOZO JM, DARTRIGUES JF. Are sex and educational level independant predictors of dementia and Alzheimer's disease ? Incidence data from the PAQUID project. J Neurol Neurosurg Psychiatry 1999;99:177-183.

11.LAURENT B, DUBOIS B, TOUCHON J, LEYS D, PASQUIER F. Critères diagnostiques des démences. Esaïe - Pfizer, Nov $2000 ; 58$ p.

12.LINDSAY J, ANDERSON L. Dementia / Alzheimer's Disease. BMC Women's Health 2004, 4:S20.

13.MONTANI C, NOUREDDINE B. Le test du "Cadran de I'horloge". Psychologie Médicale, 1990; 22(7): 625-626. 10

14.MOUANGA AM, MISSONTSA DA. Profil socio-démographique et clinique des patients observes en consultation externe de psychiatrie au Congo-Brazzaville. Médecine d'Afrique Noire, 2005;52:279783.

15.PATTERSON et al. Le traitement de la démence : conclusions ce la conférence canadienne de consensus sur la démence. JAMC, Juin 1999; 160.18p.

16.POITRENAUD J, PIETTE F, SEBBAN C, FORETTE B. Une batterie de tests psychométriques pour l'évaluation du fonctionnement cognitif chez les sujets âgés. Rev Gériatr. 1990, $15 ; 2: 57-64$.

17.ROLLAND Y, GILLETTE-GUYONNET S, NOURHASHEMI F, ANDRIEU S, CANTET C, PAYOUX P, OUSSET PJ, VELLAS B. Wandering and Alzheimer's disease type. Descriptive study. REAL.FR research program on Alzheimer's disease and management. Rev Med Interne. 2003; 24:333-338.

18.SKA B. Epidémiologie de la Maladie d'Alzheimer et autres démences au Canada. Les sélections de Médecine/Sciences 2002; 24:27-28.

19. THOMAS PH, BOUCHER C, CLEMENT J.P, HAZIF-THOMAS C. Famille, maladie d'Alzheimer et symptômes négatifs. La revue française de psychiatrie et psychologie médicale. Avril 2000; 37:3945.

20.TSUCHIHASHI T, FUJISHIMA M. Hypertension et maladie d'Alzheimer. Alzheimer Actualités 2001; 157:10-13.

21.WIMO A, WINBLAD B, AGUERO -TORRES H, VON STRAUSS E. The magnitude of démentia occurrence in the world. Alzheimer Dis Ass Disord 2003; 17(2):63-7.

22. YAMÉOGO NV. La demande de soins du sujet âgé au Centre Hospitalier Universitaire Yalgado Ouédraogo. Thèse méd. 2005; 75p. 